\title{
Prenatal polychlorinated biphenyl exposure promotes invasion of progeny ectopic endometrial stromal cells via epigenetic modification of EZH2
}

\section{Yinfeng Wang ( $\square$ wangyf1004@zju.edu.cn)}

Zhejiang University School of Medicine Women's Hospital https://orcid.org/0000-0002-4489-9658

Chaoshuang Ye

Zhejiang University School of Medicine Women's Hospital

Changchang Hu

Zhejiang University School of Medicine Women's Hospital

Pei Chen

Zhejiang University School of Medicine Women's Hospital

Chaolu Chen

Zhejiang University School of Medicine Women's Hospital

\section{Ruijin Wu}

Zhejiang University School of Medicine Women's Hospital

\section{Research Article}

Keywords: polychlorinated biphenyls, pregnancy, endometriosis, invasion, epigenetics, EZH2

Posted Date: April 1st, 2021

DOI: https://doi.org/10.21203/rs.3.rs-328521/v1

License: (c) (i) This work is licensed under a Creative Commons Attribution 4.0 International License.

Read Full License 


\section{Abstract}

Polychlorinated biphenyls(PCBs) are persistent environmental endocrine disruptor. This study aim to investigate the changes of the ectopic endometrium invasive ability and the possible mechanism after exposure to PCBs during pregnancy. In total, 12 female Sprague Dawley rats were intraperitoneally injected with Aroclor $1221(1 \mathrm{mg} / \mathrm{kg})$ or dimethyl sulfoxide $(1 \mathrm{mg} / \mathrm{kg})$ at 16 and 18 days of gestation. The endometriosis model was established by autogenous uterine abdominal wall implantation 2 months after birth. The degree of adhesion between the endometriosis and the greater omentum, as well as cell morphology and Transwell invasion patterns were used to evaluate the invasive ability of progeny ectopic endometrial stromal cells (ESCs). Moreover, the effect of the enhancer of zeste 2 polycomb repressive complex 2 subunit (EZH2)/ trimethylation of Histone 3 lysine 27 (H3K27me3) axis was examined using a highly selective EZH2 inhibitor, GSK126. After gestational PCBs exposure, the adhesion between the endometrium and the greater omentum was enhanced $(P<0.05)$, and the length and number of microvilli were significantly increased $(P<0.01)$. Exposure to $P C B s$ during pregnancy increased the invasive ability of the ectopic ESCs $(P<0.01)$, with upregulated expression levels of EZH2 and H3k27me3, which were abrogated by the EZH2 inhibitor GSK126. In conclusion, exposure to PCBs during pregnancy increased the invasive ability of the ectopic endometrium, which may be mediated by EZH2.

\section{Introduction}

Endometriosis is a chronic benign gynecologic disease in which the growing endometrium appears outside the uterus, causing chronic pelvic pain, dysmenorrhea, infertility and dysmenorrhea (Altayyeb et al. 2020). There are various theories regarding the pathogenesis of endometriosis, such as retrograde menstruation, genetic factors, blood lymphatic spread, cell apoptosis, immune factors and environmental toxins (Lagana et al. 2017; Parazzini et al. 2013; Sofo et al. 2015). Although the classic 'Retrograde menstruation' theory is widely accepted, it does not account for all cases of endometriosis(Giudice and Kao 2004; McKinnon et al. 2018). With the development of industrialization, environmental problems are prominent, and the influence of environmental endocrine disruptors on the incidence of endometriosis has attracted increased attention (Gerkowicz et al. 2020; Martinez-Zamora et al. 2015; Matta et al. 2020; Wen et al. 2020). Accumulating evidence has suggested that exposure to certain environmental endocrine chemicals, including dioxins, bisphenol A, phthalates and polychlorinated biphenyls (PCBs), may increase the risk of endometriosis (Simonelli et al. 2017; Smarr et al. 2016). Furthermore, our previous study has confirmed that PCBs are associated with the migration and invasion of ectopic endometrial stromal cells through in vitro culture of ectopic endometrial stromal cells (ESCs) (Hu et al. 2018).

PCBs are synthetic organic compounds, which contain 209 homologs. Due to their physicochemistry stability, non-flammability and favorable electrical insulation properties, PCBs were widely used in capacitors, lubricants, adhesives, turbines and plasticizers (Andersen et al. 2020; Ravenscroft et al. 2018), Although PCBs have been banned globally since the 1970s, they are still released from industrial incinerators and landfills, or leaked from old capacitors and transformers (Adams et al. 2019; Ravenscroft et al. 2018). With characteristics such as the resistance to environmental degradation, dissolving in liquid 
and bioaccumulation, PCBs can circulate in the environment via the food chain, water, soil and air (Klocke et al. 2019; Warenik-Bany et al. 2019). They also transfer from the mother to fetus via the placenta and lactation (Abraham et al. 1998). According to the hypothesis of the Developmental Origins of Health and diseases $(\mathrm{DOHaD})$, gestation is considered to be a particularly sensitive time to external environment. Intrauterine exposure to an adverse environment will not only affect fetal development and lead to unfavorable pregnancy outcomes, but it will also have long-term impacts on disease susceptibility into late adulthood (Barker 1997; Godfrey and Barker 2001), including endometriosis (Wei et al. 2016).

Previous studies have reported that human diseases are associated with epigenetic regulation caused by early life exposure to PCBs (Casati et al. 2013; Caspersen et al. 2016; Gladen et al. 2000; Su et al. 2015; Tang-Peronard et al. 2011). Histone methylation is one of the important epigenetic mechanisms, and is mediated by interacting with steroid receptors or non-genomic signaling pathways (Casati et al. 2012; Casati et al. 2013; Casati et al. 2015). Histone methyltransferase enhancer of zeste homolog 2 (EZH2) is an enzyme-catalyzed subunit of polycomb repressive complex 2 (PRC2) that alters gene expression by trimethylation of Histone 3 lysine 27 (H3K27me3)(Jones et al. 2018), which inhibits chromatin structure remodeling by switch/sucrose non-fermentable (SWI/SNF), resulting in gene inactivation. EZH2 is associated with the invasion and progression of breast cancer, lymphoma, ovarian cancer and other malignant tumors, and is consider to be one of the new targeted therapeutic factors for malignant tumors (Booth et al. 2018; Chang et al. 2011; Italiano et al. 2018; McCabe et al. 2012). Although endometriosis is a benign disease, it has biological characteristics of invasion (Zhang et al. 2017). The invasion indices of peritoneal endometriosis cells is similar to that of metastatic bladder cancer cells, while normal endometrial cells are non-invasive (Gaetje et al. 1995).

Based on previous research, we suppose that gestational exposure to PCBs increases the invasive ability of the ectopic endometrium, thus leading to the occurrence and development of endometriosis via the EZH2/ H3K27me3 pathway.

\section{Material And Methods}

Animal model. The present study was performed in the Experimental Research Laboratory of Zhejiang Chinese Medical University, and complied with the approval of the ethic committee (approval no.

2018102904). In total, 12 non-pregnant female and 12 male Sprague Dawley (SD) rats (age, 10-12 weeks; weight, 180-220 g) were purchased from SLAC Laboratory Animal Co., Ltd. Rats were housed in a single cage with an environmentally controlled temperature $\left(10-24^{\circ} \mathrm{C}\right)$ and humidity $(50-70 \%)$ under a $12 / 12 \mathrm{~h}$ (light/dark) schedule. They were feed with standard pellet food and water. The experiment began $\geq 1$ week after the rats arrived to acclimatize them to the new housing conditions.

The female rats were randomly assigned into either a PCB group $(n=6)$ or a control group $(n=6)$. Any female rat was housed with a random male rat. The successful mating (indicated by morning sperm positive vaginal smear) was termed embryonic day (E)0. On E16 and E18, the rats in PCB group and control group were intraperitoneal injected with $1 \mathrm{mg} / \mathrm{kg}$ Aroclor1221 (cat. no. C221N; AccuStandard; New 
Haven, CT) or dimethyl sulfoxide (DMSO, cat. no. D4540; Sigma-Aldrich; Merck KGaA), respectively, as previously reported (Bell et al. 2016; Reilly et al. 2015; Steinberg et al. 2007; Steinberg et al. 2008; Walker et al. 2013).

Male pups were removed after delivery. The endometriosis model was performed 2 months postpartum as described by Saltan et al (Saltan et al. 2016). The rats were fixed in the supine position after general anesthesia with intraperitoneally injected $3 \%$ chloral hydrate (350 mg/kg). A 3-4-cm longitudinal incision was made in the middle of the lower abdomen. The left side of uterine cornu was excised and cut into $5 \times 5 \mathrm{~mm}$ pieces. The endometrium layer was stripped from myometrium and autotransplanted at the abdominal walls with abundant blood supply (Fig. 1). In total, two pieces of endometrium were transplanted on each side. The abdominal cavity was closed with absorbable suture. A single intramuscular injection of $80,000 \mathrm{U}$ penicillin was administered to prevent infection.

The rats were anesthetized, as previously mentioned, 1 month after the first operation and were surgically examined to visualize the adhesion score of endometriotic foci. The adhesion scored was blindly evaluated by two doctors as previously reported by Belluco et al (Belluco et al. 2001), which involved the extent of site involvement (0-4), type of adhesion (0-4) and the tenacity of adhesion (0-3). The endometriotic foci were cultured or immobilized by $10 \%$ formalin for further experiment. Then, rats were euthanized by carbon dioxide inhalation.

Cell culture and treatment. The ectopic endometrium was cut into pieces and then digested with $0.2 \%$ type I collagenase (Sigma-Aldrich; Merck KGaA) for $1 \mathrm{~h}$ on a horizontal concussion meter at $37^{\circ} \mathrm{C}$ and 180 rpm. After centrifugation at 1,000 rpm for $5 \mathrm{~min}, 3-5 \mathrm{ml}$ PBS was added and the cell suspension was filtered with 100 and $40 \mu \mathrm{m}$ strainers. Cells were centrifuged at 1,000 rpm for $5 \mathrm{~min}$ at $37^{\circ} \mathrm{C}$, then an appropriate amount of DMEM/F12 medium was added and incubated in $5 \% \mathrm{CO} 2$ at $37^{\circ} \mathrm{C}$ in an incubator (Forma 3111; Thermo Fisher Scientific, Inc.). The culture medium was replaced at $24 \mathrm{~h}$ after primary culture and then every 2-3 days.

In PCB group, GSK126 (cat. no.5005800001, Sigma-Aldrich; Merck KGaA), a highly selective inhibitor of EZH2 via competitive combination with S-adenosine methionine (McCabe et al. 2012), was added to evaluate the role of EZH2. ESCs were inoculated into a 6-well plate with a density of $3.0 \times 10^{5}$ per well, and GSK126 was added at the doses of $0,2.5,5,10$ and $20 \mu \mathrm{M}$ for $48 \mathrm{~h}$, where $5 \mu \mathrm{M}$ was the optimal inhibitory amount and was used in subsequent experiments. GSK126 was not added to the control group. The role of EZH2 in ESCs invasion was further determined by observing microvilli under transmission electron microscopy and transwell invasion assay.

Immunofluorescence. The purity of ESCs was determined via immunofluorescence, using Cytokeratin 7(cat. no. ab181598, Abcam) and Vimentin(cat. no. ab92547, Abcam) as markers of epithelial and stromal cells respectively. The primary cells were fixed with $4 \%$ paraformaldehyde at room temperature for 30 min, followed by incubation with $0.5 \%$ tritox-100 at room temperature for $15 \mathrm{~min}$. Cells were then washed three times with PBS and sealed with $5 \%$ BSA(Amresco, USA) solution at $37^{\circ} \mathrm{C}$ for $1 \mathrm{~h}$. 
Subsequently, anti-vimentin antibody or Cytokeratin 7 were added, incubated at $4^{\circ} \mathrm{C}$ overnight and washed three times with PBS. FITC-labeled IgG(Amresco, USA) was added at room temperature for $1 \mathrm{~h}$ and cells were washed three times with PBS. Finally, $2 \mathrm{mg} / \mathrm{I}$ DAPI solution was added to re-stain the nucleus at room temperature for $10 \mathrm{~min}$. Cells were washed twice with PBS and sealed with neutral resin, and the fluorescence signals of the cells were observed under a fluorescence microscope (Axiovert200; Zeiss AG).

Transmission electron microscopy. Transmission electron microscopy was performed as described previously (Chen et al. 2019). ESCs cells were fixed overnight with $2.5 \%$ glutaraldehyde at $4^{\circ} \mathrm{C}$ and then fixed with $1 \%$ osmium acid for $1 \mathrm{~h}$ at room temperature. Cells were rinsed with water 2-3 times for 10 min. Then, 50, 70, 90 and $100 \%$ ethanol for 15 min and $100 \%$ acetone for $15-20$ min were used for dehydration at room temperature. Cells were embedded at room temperature for $4 \mathrm{~h}$ and ultrathin sections at 70-nm were sliced. The sections were placed in copper grids and stained with uranyl acetate and lead citrate. A transmission electron microscope (FEI Tecnai T10, USA) was used to observe the number and length of microvilli. In total, five fields were randomly selected from each section. The length of microvilli was measured using Image J software (V1.51, National Institute of Health). The number of microvilli was counted as number/circumference $(n / \mu m)$.

Immunohistochemistry. All tissues were fixed with $10 \%$ neutral formalin, paraffin-embedded and prepared into slices of 4- $\mu \mathrm{m}$ thickness. The process and scoring of immunohistochemistry were based on a previous report (Kumari et al. 2018). According to the antibody instructions, each slice was incubated with 50-100 $\mu \mathrm{M}$ primary antibody and the tissue solution (EZH2, 1:8,000, cat. no. ab191080; H3K27me3, 1:40, cat. no. ab6002; Abcam) overnight at $4^{\circ} \mathrm{C}$. Then, 50-100 $\mu$ l Envision $+/$ horseradish peroxidase (HRP) secondary antibody (DAKO; Agilent Technologies, Inc.) was added to each slice and incubated at room temperature for 30-60 min. The immunoreactivity was detected using diaminobenzidine. The slices were subsequently stained with hematoxylin, and observed under a light microscope (DM500; Leica Microsystems $\mathrm{GmbH}$ ), and images were captured at a magnification of $x 40$ or $\times 200$. The staining intensity of ESCs was scored as follows: 1 for absent or weak; 2 for moderate; and 3 for strong. The percentage positive cells was scored as: Percent of cells $<1,0$ point; percent of cells between $1-10,1$ point; percent of cells between 11-33, 2 points; percent of cells between 34-66, 3 points; and percent of cells between $67-100,4$ points. The $Q$ score of all the slices was calculated by multiplying the staining intensity by the percentage positive of ECSs cells (Kumari et al. 2018).

Western blotting. Western blotting was performed as described previously (Chen et al. 2019). ESCs were washed in PBS prior to being lysed in RIPA lysis buffer (Beyotime Institute of Biotechnology). The proteins were electrophoresed via 12\% SDS-PAGE and transferred to PVDF membranes(Millipore, Billerica, MA, USA). Membranes were blocked in $3 \%$ bovine serum albumin (BSA, Sigma-Aldrich; Merck KGaA) at $37^{\circ} \mathrm{C}$ for $2 \mathrm{~h}$ and then incubated with primary antibodies (EZH2, 1:100; H3K27me3, 1:80) overnight at $4^{\circ} \mathrm{C}$. Then, membranes were incubated with horseradish peroxidase-conjugated secondary antibody $(1: 10,000$, cat.no. ab236555, Abcam) for $1.5 \mathrm{~h}$ at room temperature. Enhanced chemiluminescence detection reagent (Sigma-Aldrich, St Louis, MO, USA) was used to detect the signal. Relative protein expression 
levels were semi-quantified with $\beta$-actin expression as the reference gene using ImageJ software (V1.51, National Institute of Health).

Reverse transcription-quantitative PCR (RT-qPCR). EZH2 gene expression was analyzed using RT-qPCR with the following sequences (Sangon Biotech Co., Ltd.) as previously reported (Chen et al. 2019): EZH2 forward, 5'-CTTACTGCTGGCACCGTCTG-3' and reverse, 5'-CTTTCCCTCTTCTGTCTGCTTCA-3'; and GAPDH forward, 5'- CTGGAGAAACCTGCCAAGTATG-3' and reverse, 5'- GGTGGAAGAATGGGAGTTGCT-3'. Total mRNA was extracted from ESCs using RNAiso Plus reagent TaKaRa RNAiso Plus (Takara Bio, Inc.) according to the manufacturer's instructions. Total RNA was reverse transcribed to cDNA using the PrimeScript ${ }^{\text {TM }}$ RT reagent (Takara Bio, Inc.). qPCR was performed using a SYBR Premix Ex Taq ${ }^{\text {TM }} \otimes$ kit $^{2}$ (Takara Bio, Inc.) according to the manufacturer's instructions. The following thermocycling conditions were used: Amplification of $48 \mathrm{cycles}$ of $95^{\circ} \mathrm{C}$ for $10 \mathrm{sec}, 60^{\circ} \mathrm{C}$ for $30 \mathrm{sec}$. The cyclic threshold (Cq) value was calculated using the average of three wells for each sample. The relative expression levels of target genes were normalized against actin using the $2^{-\Delta \Delta C q}$ method(Livak and Schmittgen 2001).

Cell migration and invasion assays. The migration and invasion of ESCs were evaluated as previously reported (Hu et al. 2018). Transwell chambers were 6.5-mm in diameter with an 8- $\mu \mathrm{m}$ pore size (Corning, Inc.). A density of $2 \times 10^{5}$ cells with $200 \mu$ l serum-free DMEM/F12 was seeded on the upper chamber. The lower chamber was filled with $500 \mu \mathrm{l}$ DMEM/F12 containing 10\% FBS. After incubation for $24 \mathrm{~h}$, the cells on the underside of the membrane were fixed and then stained with crystal violet solution. For the invasion assay, the upper chamber was pre-coated with $25 \mu \mathrm{l}$ Matrigel (BD Biosciences), and incubated for $48 \mathrm{~h}$. A total of five fields were randomly selected to count the number of cells under a light microscope (x100).

Statistical analysis. Statistical analyses were performed using SPSS 23.0 software (IBM Corp.). Data are presented as the mean \pm SD. Student's t-test was used to analyze differences for paired data and one-way ANOVA was used to analyze multiple comparisons. $P<0.05$ was considered to indicate a statistically significant difference.

\section{Results}

Identification of endometriosis and ESCs. The PCB group had 23 female offsprings, while the control group had 25( $P>0.05)$. One month after the endometrium transplantation, the endometriotic implants formed a cystic nodule with vascularization. Hematoxylin and eosin staining showed that the ectopic lesions formed one or several cysts, and the cyst wall was composed of epithelial cell layer, stromal cell layer and a small amount of fibrous connective tissue from the inside to the outside. After 2-4 generations of purification, the purity of ESCs was $>95 \%$. It had a long spindle shape with an oval nucleus, and immunofluorescence detection identified that Vimentin was positive and Cytokeratin7 was negative (Fig. 2).The adhesion score of the control group was 4.6 \pm 1.3 . After PCB exposure during pregnancy, the adhesion score of ectopic endometrial lesions in the offspring was significantly increased to $7.1 \pm 0.9(P<0.05)$. 
PCB promotes the invasion of offspring ectopic ESCs and GSK126 abrogates the effect. There was no difference in the migratory ability of the ESCs from rat offspring. However, the invasive potential was significantly increased in PCB group compared with the control group. To investigate the effect of EZH2 on cell invasion, ESCs were treated with GSK126 for $48 \mathrm{~h}$. In addition, after treatment with GSK126, it was proved that the down-regulation of EZH2 significantly reduced the invasion ability of ESCs(Fig. 3).

$P C B$ exposure increases the length and number of microvilli via EZH2. Transmission electron microscope was used to assess the microvilli on the surface of ESCs. The microvilli were mainly slender cylindrical and interwoven with the surrounding microvilli, and their bases were connected to the cell membrane. The number of microvilli in the PCB exposed group was $1.07 \pm 0.21$ per perimeter $(\mu \mathrm{m})$, and that in the control group was $0.67 \pm 0.26$ per perimeter $(\mu \mathrm{m})(P<0.01)$. Moreover, the microvilli length was $1.14 \pm 0.58 \mu \mathrm{m}$ in PCB group and $0.82 \pm 0.32 \mu \mathrm{m}$ in control group, which demonstrated a significant difference $(P<0.01)$.

After GSK126 treatment, the microvilli were smooth and the length was shortened. The number of microvilli was $0.43 \pm 0.21$ per perimeter $(\mu \mathrm{m})$ and the length of microvilli was $0.67 \pm 0.37 \mu \mathrm{m}$, which were significantly reduced compared with those of the PCB group (Fig. 4).

The expression of EZH2 and H3K27me3 increased in offspring ectopic ESCs. After exposure to PCBs during pregnancy, the expression levels of EZH2 and H3k27me3 in the ectopic ESCs of the offspring were significantly increased $(P<0.01)$. In the cell culture of the PCB group, after adding the inhibitor GSK126, Western blotting detected that the expression levels of EZH2 and its target molecule H3K27me3 were significantly decreased(Fig. 5).

\section{Discussion}

Endometriosis is a common refractory gynecological disease. Although it is estrogen-dependent benign disease, its biological behavior is similar to that of malignant tumors (Zhang et al. 2017). The migration, adhesion and invasion of retrograde endometrial cells are important steps in the formation of ectopic lesions (Gaetje et al. 1995; Meng et al. 2019; Witz et al. 2001). Previous studies have reported that PCBs increase the invasion of ESCs, as well as the incidence of endometriosis (Hu et al. 2018; Ploteau et al. 2016; Ploteau et al. 2017; Roy et al. 2015). However, little is known regarding the relationship between gestational PCBs in offspring endometriosis, and the role of histone methylation is not fully understood. In the present study, it was found that prenatal exposure to PCBs significantly increase the invasive ability of offspring ectopic ESCs with high expression levels of EZH2 and H3K27me3, which serve an important role in promoting this proses.

An increase in the number and length of microvilli was the main morphological change observe in the present study. The relationship between cell morphology and cell invasiveness has been widely studied in tumors (Antonescu and Baren 2004; Aznavoorian et al. 1993). The ultrastructure of the cell surface can predict the contact, movement and adhesion of cells to other cells and the cell matrix, which serves an important role in the occurrence and development of tumors (Black 1980; Nicolson and Poste 1976). A decrease of tumor cell invasiveness is often accompanied by the loss of microvilli (Priester et al. 2013), 
while more microvilli and pseudopodia on the cell surface indicates a higher invasive ability of tumor cells (Yan et al. 2013). In patients with endometriosis, the morphology of endometrial gland epithelial cells (EGECs) is irregular, the number and length of microvilli is increased, the organelles are abundant and EGECs possess proliferative, migratory and invasive abilities, which are associated with the formation of endometriosis cysts (Yu et al. 2018).

It has been reported that Aroclor1221 is a mildly chlorinated PCBs mixture, and its level is difficult to determine directly in the offspring after gestational exposure (Thomas et al. 1998). According to previous literature reports (Bell et al. 2016; Reilly et al. 2015; Steinberg et al. 2007; Steinberg et al. 2008; Walker et al. 2013), the dose of $1 \mathrm{mg} / \mathrm{kg}$ intraperitoneal injection of Aroclor1221 at E16 and E18 is close to the observed exposure of humans and wild animals in PCBs contaminated areas. PCBs may inhibit the activity of human estrogen sulfotransferase, an estrogen-degrading enzyme (Kester et al. 2000), thereby increasing the secretion of estrogen and the risk of hormone-dependent diseases. Accumulating evidence had suggested that higher PCBs exposure may increase the risk of endometriosis (Hu et al. 2018; Martinez-Zamora et al. 2015; Porpora et al. 2006; Wei et al. 2016). A reduced responsiveness to progesterone and overexpression of matrix metalloproteinases may be a key element of endometrial dysfunction associated with endometriosis (Osteen et al. 2005). With similar effects to 2,3,7,8tetrachlorodibenzo-p-dioxin (TCDD), dioxin-like-PCBs can combine with aryl hydrocarbon receptor(AhR) and form an activated heterodimerization complex with the aromatic hydrocarbon receptor nuclear translocator protein, leading to the overexpression of AhR in endometrioid cells (Okino and Whitlock 2000; Sherr and Monti 2013). Over activated AhR induced by PCBs regulates multiple downstream biological events, including the epigenetic silencer activity of histone methyltransferase EZH2 (Lee et al. 2020).

Epigenetics refers to the heritable non-gene sequence changes, including DNA methylation, posttranslational histone modification and microRNA. However, these are dynamic and may be affected by environmental exposures (Holland 2017). Pregnancy is a critical period of fetal epigenetic remodeling, which is sensitive to environmental endocrine disruptors (Casati et al. 2015; Pozharny et al. 2010). Maternal health can determine susceptibility to certain diseases in adulthood (Kanherkar et al. 2014). Moreover, gestational PCBs exposure not only induces an aberrant DNA methylation pattern, but also affect the histone modification balance, leading to long term and transgenerational effects (Casati et al. 2015).

Histone methylation has been shown to serve an important role in the etiology of endometriosis, and EZH2 and H3K27me3 are epigenetic marks of endometriosis (Colon-Caraballo et al. 2015). Zhang et al (Zhang et al. 2017) revealed that EZH2 was associated with the invasion of the ectopic endometrium, and that the levels of EZH2 and $\mathrm{H} 3 \mathrm{~K} 27 \mathrm{me} 3$ in the ectopic endometrium were significantly higher compared with those in normal endometrium. EZH2 and H3K27me3 directly induce epithelialmesenchymal transition, thereby promoting the occurrence and development of endometriosis (Cao et al. 2008; Colon-Caraballo et al. 2015; Zhang et al. 2017). The present study also identified similar findings; after PCBs exposure during pregnancy, the expression levels of EZH2 and H3K27me3 in ectopic ESCs were significantly increased and the cell invasive ability was enhanced. However, after inhibition of EZH2 
using GSK126, the expression levels of EZH2 and H3K27me3 were decreased, accompanied by a decreased cell invasive ability, suggesting that $\mathrm{EZH} 2$ serves an important role in ectopic endometrial invasion caused by PCBs exposure during pregnancy.

In conclusion, PCBs exposure during pregnancy serves a significant role in the development and progression of endometriosis in offspring. As an early stage of life development, women should consider the effects of environmental pollution during pregnancy with regards to potential harm to next generations. EZH2-mediated epigenetic regulation may serve an important role in this process. However, further research is required to identify the underlying molecular mechanisms activated by EZH2 and H3K27me3.

\section{Declarations}

\section{Ethics approval and consent to participate}

This study was approved by the Ethics Committee of Zhejiang Chinese Medical University (approval no. 2018102904).

\section{Patient consent for publication}

Not applicable.

\section{Availability of data and materials}

All data generated or analyzed during this study are included in this published article.

\section{Competing interests}

The authors declare that they have no competing interests.

\section{Funding}

This study was funded by a grant from the Zhejiang Provincial Key Research \& Development Project Grants (grant no.2021C03095) and Education Department of Zhejiang Province (grant no. 2019150).

\section{Authors' contributions}

WYF performed the experiments $\llbracket \mathrm{HCC}$ wrote the manuscript, YCS performed the experiments, CP and CCLanalyzed the data. WRJ designed the study. All authors read and approved the final manuscript.

\section{Acknowledgements}

Not applicable.

\section{References}


Abraham K, Papke O, Gross A, Kordonouri O, Wiegand S, Wahn U, Helge H (1998) Time course of PCDD/PCDF/PCB concentrations in breast-feeding mothers and their infants Chemosphere 37:1731-1741

Adams EM, von Hippel FA, Hungate BA, Buck CL (2019) Polychlorinated biphenyl (PCB) contamination of subsistence species on Unalaska Island in the Aleutian Archipelago Heliyon 5:e02989 doi:10.1016/j.heliyon.2019.e02989

Altayyeb A et al. (2020) Characterization of Mechanical Signature of Eutopic Endometrial Stromal Cells of Endometriosis Patients Reprod Sci doi:10.1007/s43032-019-00042-3

Andersen HV, Gunnarsen L, Knudsen LE, Frederiksen M (2020) PCB in air, dust and surface wipes in 73 Danish homes Int J Hyg Environ Health:113429 doi:10.1016/j.ijheh.2019.113429

Antonescu CR, Baren A (2004) Spectrum of low-grade fibrosarcomas: a comparative ultrastructural analysis of low-grade myxofibrosarcoma and fibromyxoid sarcoma Ultrastruct Pathol 28:321-332 doi:10.1080/019131290882259

Aznavoorian S, Murphy AN, Stetler-Stevenson WG, Liotta LA (1993) Molecular aspects of tumor cell invasion and metastasis Cancer 71:1368-1383 doi:10.1002/1097-0142(19930215)71:4<1368::aidcncr2820710432>3.0.co;2-I

Barker DJ (1997) Fetal nutrition and cardiovascular disease in later life Br Med Bull 53:96-108 doi:10.1093/oxfordjournals.bmb.a011609

Bell MR, Hart BG, Gore AC (2016) Two-hit exposure to polychlorinated biphenyls at gestational and juvenile life stages: 2. Sex-specific neuromolecular effects in the brain Mol Cell Endocrinol 420:125-137 doi:10.1016/j.mce.2015.11.024

Belluco C et al. (2001) Prevention of postsurgical adhesions with an autocrosslinked hyaluronan derivative gel J Surg Res 100:217-221 doi:10.1006/jsre.2001.6248

Black PH (1980) Shedding from the cell surface of normal and cancer cells Adv Cancer Res 32:75-199 doi:10.1016/s0065-230x(08)60361-9

Booth CAG et al. (2018) Ezh2 and Runx1 Mutations Collaborate to Initiate Lympho-Myeloid Leukemia in Early Thymic Progenitors Cancer Cell 33:274-291 e278 doi:10.1016/j.ccell.2018.01.006

Cao Q et al. (2008) Repression of E-cadherin by the polycomb group protein EZH2 in cancer Oncogene 27:7274-7284 doi:10.1038/onc.2008.333

Casati L, Sendra R, Colciago A, Negri-Cesi P, Berdasco M, Esteller M, Celotti F (2012) Polychlorinated biphenyls affect histone modification pattern in early development of rats: a role for androgen receptordependent modulation? Epigenomics 4:101-112 doi:10.2217/epi.11.110 
Casati L, Sendra R, Poletti A, Negri-Cesi P, Celotti F (2013) Androgen receptor activation by polychlorinated biphenyls: epigenetic effects mediated by the histone demethylase Jarid1b Epigenetics 8:1061-1068 doi:10.4161/epi.25811

Casati L, Sendra R, Sibilia V, Celotti F (2015) Endocrine disrupters: the new players able to affect the epigenome Front Cell Dev Biol 3:37 doi:10.3389/fcell.2015.00037

Caspersen IH et al. (2016) Maternal dietary exposure to dioxins and polychlorinated biphenyls (PCBs) is associated with language delay in 3year old Norwegian children Environ Int 91:180-187 doi:10.1016/j.envint.2016.02.031

Chang CJ et al. (2011) EZH2 promotes expansion of breast tumor initiating cells through activation of RAF1-beta-catenin signaling Cancer Cell 19:86-100 doi:10.1016/j.ccr.2010.10.035

Chen C, Zhou Y, Hu C, Wang Y, Yan Z, Li Z, Wu R (2019) Mitochondria and oxidative stress in ovarian endometriosis Free Radic Biol Med 136:22-34 doi:10.1016/j.freeradbiomed.2019.03.027

Colon-Caraballo M, Monteiro JB, Flores I (2015) H3K27me3 is an Epigenetic Mark of Relevance in Endometriosis Reprod Sci 22:1134-1142 doi:10.1177/1933719115578924

Gaetje R, Kotzian S, Herrmann G, Baumann R, Starzinski-Powitz A (1995) Invasiveness of endometriotic cells in vitro Lancet 346:1463-1464 doi:10.1016/s0140-6736(95)92474-4

Gerkowicz SA et al. (2020) Endometriosis, endocrine disrupters, and epigenetics: an investigation into the complex interplay in women with polybrominated biphenyl exposure and endometriosis $\mathrm{J}$ Assist Reprod Genet doi:10.1007/s10815-020-01695-9

Giudice LC, Kao LC (2004) Endometriosis Lancet 364:1789-1799 doi:10.1016/S0140-6736(04)17403-5

Gladen BC, Ragan NB, Rogan WJ (2000) Pubertal growth and development and prenatal and lactational exposure to polychlorinated biphenyls and dichlorodiphenyl dichloroethene J Pediatr 136:490-496 doi:10.1016/s0022-3476(00)90012-x

Godfrey KM, Barker DJ (2001) Fetal programming and adult health Public Health Nutr 4:611-624 doi:10.1079/phn2001145

Holland N (2017) Future of environmental research in the age of epigenomics and exposomics Rev Environ Health 32:45-54 doi:10.1515/reveh-2016-0032

Hu T, Yao M, Fu X, Chen C, Wu R (2018) Polychlorinated biphenyl 104 promotes migration of endometrial stromal cells in endometriosis Toxicol Lett 290:19-28 doi:10.1016/j.toxlet.2018.03.009

Italiano A et al. (2018) Tazemetostat, an EZH2 inhibitor, in relapsed or refractory B-cell non-Hodgkin lymphoma and advanced solid tumours: a first-in-human, open-label, phase 1 study Lancet Oncol 19:649- 
Jones BA, Varambally S, Arend RC (2018) Histone Methyltransferase EZH2: A Therapeutic Target for Ovarian Cancer Mol Cancer Ther 17:591-602 doi:10.1158/1535-7163.MCT-17-0437

Kanherkar RR, Bhatia-Dey N, Csoka AB (2014) Epigenetics across the human lifespan Front Cell Dev Biol 2:49 doi:10.3389/fcell.2014.00049

Kester MH et al. (2000) Potent inhibition of estrogen sulfotransferase by hydroxylated PCB metabolites: a novel pathway explaining the estrogenic activity of PCBs Endocrinology 141:1897-1900 doi:10.1210/endo.141.5.7530

Klocke C, Sethi S, Lein PJ (2019) The developmental neurotoxicity of legacy vs. contemporary polychlorinated biphenyls (PCBs): similarities and differences Environ Sci Pollut Res Int doi:10.1007/s11356-019-06723-5

Kumari K, Das B, Adhya A, Chaudhary S, Senapati S, Mishra SK (2018) Nicotine associated breast cancer in smokers is mediated through high level of EZH2 expression which can be reversed by methyltransferase inhibitor DZNepA Cell Death Dis 9:152 doi:10.1038/s41419-017-0224-z

Lagana AS et al. (2017) Unus pro omnibus, omnes pro uno: A novel, evidence-based, unifying theory for the pathogenesis of endometriosis Med Hypotheses 103:10-20 doi:10.1016/j.mehy.2017.03.032

Lee JE, Cho SG, Ko SG, Ahrmad SA, Puga A, Kim K (2020) Regulation of a long noncoding RNA MALAT1 by aryl hydrocarbon receptor in pancreatic cancer cells and tissues Biochem Biophys Res Commun 532:563-569 doi:10.1016/j.bbrc.2020.08.053

Livak KJ, Schmittgen TD (2001) Analysis of relative gene expression data using real-time quantitative PCR and the 2(-Delta Delta C(T)) Method Methods 25:402-408 doi:10.1006/meth.2001.1262

Martinez-Zamora MA et al. (2015) Increased levels of dioxin-like substances in adipose tissue in patients with deep infiltrating endometriosis Hum Reprod 30:1059-1068 doi:10.1093/humrep/dev026

Matta K et al. (2020) Associations between persistent organic pollutants and endometriosis: A multipollutant assessment using machine learning algorithms Environ Pollut 260:114066 doi:10.1016/j.envpol.2020.114066

McCabe MT et al. (2012) EZH2 inhibition as a therapeutic strategy for lymphoma with EZH2-activating mutations Nature 492:108-112 doi:10.1038/nature11606

McKinnon B, Mueller M, Montgomery G (2018) Progesterone Resistance in Endometriosis: an Acquired Property? Trends Endocrinol Metab 29:535-548 doi:10.1016/j.tem.2018.05.006 
Meng X, Liu J, Wang H, Chen P, Wang D (2019) MicroRNA-126-5p downregulates BCAR3 expression to promote cell migration and invasion in endometriosis Mol Cell Endocrinol 494:110486 doi:10.1016/j.mce.2019.110486

Nicolson GL, Poste G (1976) The cancer cell: dynamic aspects and modifications in cell-surface organization (first of two parts) N Engl J Med 295:197-203 doi:10.1056/NEJM197607222950405

Okino ST, Whitlock JP, Jr. (2000) The aromatic hydrocarbon receptor, transcription, and endocrine aspects of dioxin action Vitam Horm 59:241-264 doi:10.1016/s0083-6729(00)59009-8

Osteen KG, Bruner-Tran KL, Eisenberg E (2005) Reduced progesterone action during endometrial maturation: a potential risk factor for the development of endometriosis Fertil Steril 83:529-537 doi:10.1016/j.fertnstert.2004.11.026

Parazzini F, Vigano P, Candiani M, Fedele L (2013) Diet and endometriosis risk: a literature review Reprod Biomed Online 26:323-336 doi:10.1016/j.rbmo.2012.12.011

Ploteau S, Antignac JP, Volteau C, Marchand P, Venisseau A, Vacher V, Le Bizec B (2016) Distribution of persistent organic pollutants in serum, omental, and parietal adipose tissue of French women with deep infiltrating endometriosis and circulating versus stored ratio as new marker of exposure Environ Int 97:125-136 doi:10.1016/j.envint.2016.08.011

Ploteau S et al. (2017) Associations between internal exposure levels of persistent organic pollutants in adipose tissue and deep infiltrating endometriosis with or without concurrent ovarian endometrioma Environ Int 108:195-203 doi:10.1016/j.envint.2017.08.019

Porpora MG et al. (2006) Increased levels of polychlorobiphenyls in Italian women with endometriosis Chemosphere 63:1361-1367 doi:10.1016/j.chemosphere.2005.09.022

Pozharny Y et al. (2010) Genomic loss of imprinting in first-trimester human placenta Am J Obstet Gynecol 202:391 e391-398 doi:10.1016/j.ajog.2010.01.039

Priester M et al. (2013) STAT3 silencing inhibits glioma single cell infiltration and tumor growth Neuro Oncol 15:840-852 doi:10.1093/neuonc/not025

Ravenscroft J, Schell LM, Akwesasne Task Force on the E (2018) Patterns of PCB exposure among Akwesasne adolescents: The role of dietary and inhalation pathways Environ Int 121:963-972 doi:10.1016/j.envint.2018.05.005

Reilly MP, Weeks CD, Topper VY, Thompson LM, Crews D, Gore AC (2015) The effects of prenatal PCBs on adult social behavior in rats Horm Behav 73:47-55 doi:10.1016/j.yhbeh.2015.06.002

Roy D et al. (2015) Integrated Bioinformatics, Environmental Epidemiologic and Genomic Approaches to Identify Environmental and Molecular Links between Endometriosis and Breast Cancer Int J Mol Sci 
Saltan G et al. (2016) Viburnum opulus L.: A remedy for the treatment of endometriosis demonstrated by rat model of surgically-induced endometriosis J Ethnopharmacol 193:450-455 doi:10.1016/j.jep.2016.09.029

Sherr DH, Monti S (2013) The role of the aryl hydrocarbon receptor in normal and malignant B cell development Semin Immunopathol 35:705-716 doi:10.1007/s00281-013-0390-8

Simonelli A et al. (2017) Environmental and occupational exposure to bisphenol A and endometriosis: urinary and peritoneal fluid concentration levels Int Arch Occup Environ Health 90:49-61 doi:10.1007/s00420-016-1171-1

Smarr MM, Kannan K, Buck Louis GM (2016) Endocrine disrupting chemicals and endometriosis Fertil Steril 106:959-966 doi:10.1016/j.fertnstert.2016.06.034

Sofo V et al. (2015) Correlation between dioxin and endometriosis: an epigenetic route to unravel the pathogenesis of the disease Arch Gynecol Obstet 292:973-986 doi:10.1007/s00404-015-3739-5

Steinberg RM, Juenger TE, Gore AC (2007) The effects of prenatal PCBs on adult female paced mating reproductive behaviors in rats Horm Behav 51:364-372 doi:10.1016/j.yhbeh.2006.12.004

Steinberg RM, Walker DM, Juenger TE, Woller MJ, Gore AC (2008) Effects of perinatal polychlorinated biphenyls on adult female rat reproduction: development, reproductive physiology, and second generational effects Biol Reprod 78:1091-1101 doi:10.1095/biolreprod.107.067249

Su PH, Chen HY, Chen SJ, Chen JY, Liou SH, Wang SL (2015) Thyroid and growth hormone concentrations in 8-year-old children exposed in utero to dioxins and polychlorinated biphenyls $\mathrm{J}$ Toxicol Sci 40:309-319 doi:10.2131/jts.40.309

Tang-Peronard JL, Andersen HR, Jensen TK, Heitmann BL (2011) Endocrine-disrupting chemicals and obesity development in humans: a review Obes Rev 12:622-636 doi:10.1111/j.1467-789X.2011.00871.x

Thomas GO, Sweetman AJ, Parker CA, Kreibich H, Jones KC (1998) Development and validation of methods for the trace determination of PCBs in biological matrices Chemosphere 36:2447-2459 doi:10.1016/s0045-6535(97)10211-9

Walker DM, Kermath BA, Woller MJ, Gore AC (2013) Disruption of reproductive aging in female and male rats by gestational exposure to estrogenic endocrine disruptors Endocrinology 154:2129-2143 doi:10.1210/en.2012-2123

Warenik-Bany M, Maszewski S, Mikolajczyk S, Piskorska-Pliszczynska J (2019) Impact of environmental pollution on PCDD/F and PCB bioaccumulation in game animals Environ Pollut 255:113159 doi:10.1016/j.envpol.2019.113159 
Wei M, Chen X, Zhao Y, Cao B, Zhao W (2016) Effects of Prenatal Environmental Exposures on the Development of Endometriosis in Female Offspring Reprod Sci 23:1129-1138 doi:10.1177/1933719116630418

Wen X et al. (2020) Bisphenol A Exposure Enhances Endometrial Stromal Cell Invasion and Has a Positive Association with Peritoneal Endometriosis Reprod Sci doi:10.1007/s43032-019-00076-7

Witz CA, Thomas MR, Montoya-Rodriguez IA, Nair AS, Centonze VE, Schenken RS (2001) Short-term culture of peritoneum explants confirms attachment of endometrium to intact peritoneal mesothelium Fertil Steril 75:385-390 doi:10.1016/s0015-0282(00)01699-x

Yan W, Chen Y, Yao Y, Zhang H, Wang T (2013) Increased invasion and tumorigenicity capacity of CD44+/CD24- breast cancer MCF7 cells in vitro and in nude mice Cancer Cell Int 13:62 doi:10.1186/14752867-13-62

Yu CX et al. (2018) Correlation between replicative senescence of endometrial gland epithelial cells in shedding and non-shedding endometria and endometriosis cyst during menstruation Gynecol Endocrinol 34:981-986 doi:10.1080/09513590.2018.1480709

Zhang Q, Dong P, Liu X, Sakuragi N, Guo SW (2017) Enhancer of Zeste homolog 2 (EZH2) induces epithelial-mesenchymal transition in endometriosis Sci Rep 7:6804 doi:10.1038/s41598-017-06920-7

\section{Figures}
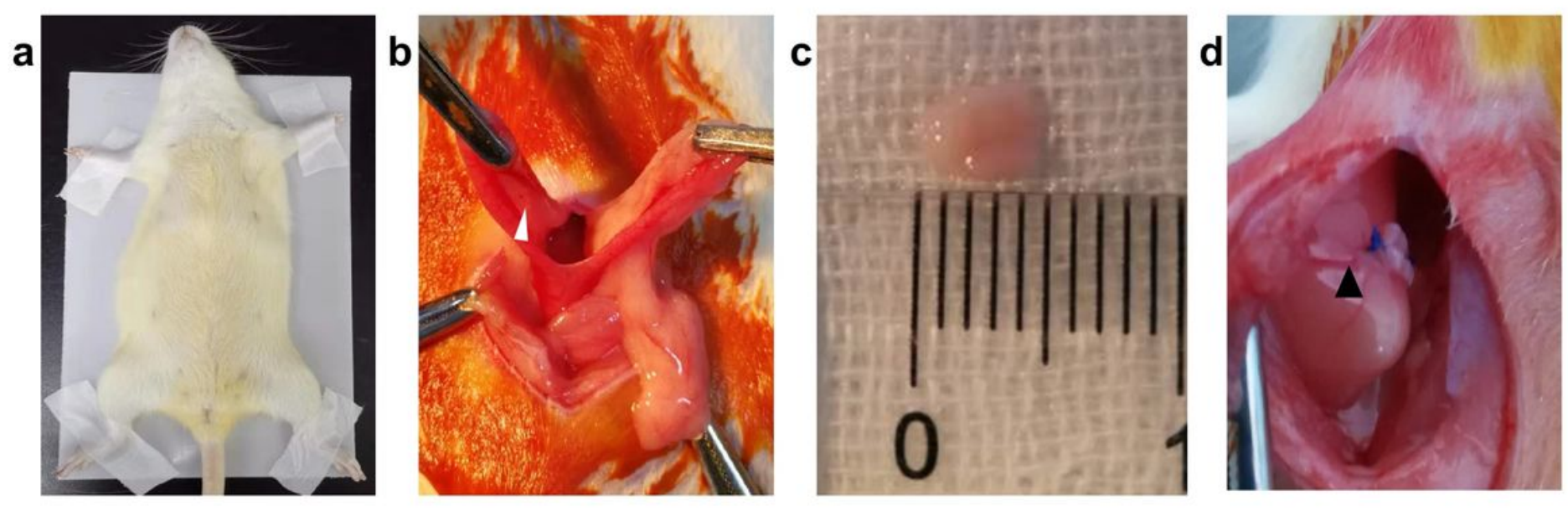

\section{Figure 1}

Rat model construction. (a) Adult female Sprague Dawley rats were fixed in supine position after anesthesia. (b) The left side of the uterine body was removed (white arrow), (c) cut into a size of $\sim 5 \times 5$ $\mathrm{mm}$ and stripped of the myometrium. (d) Autologous transplantation was performed in the area with abundant blood vessels in the abdominal wall (black arrow) to establish the endometriosis model. 

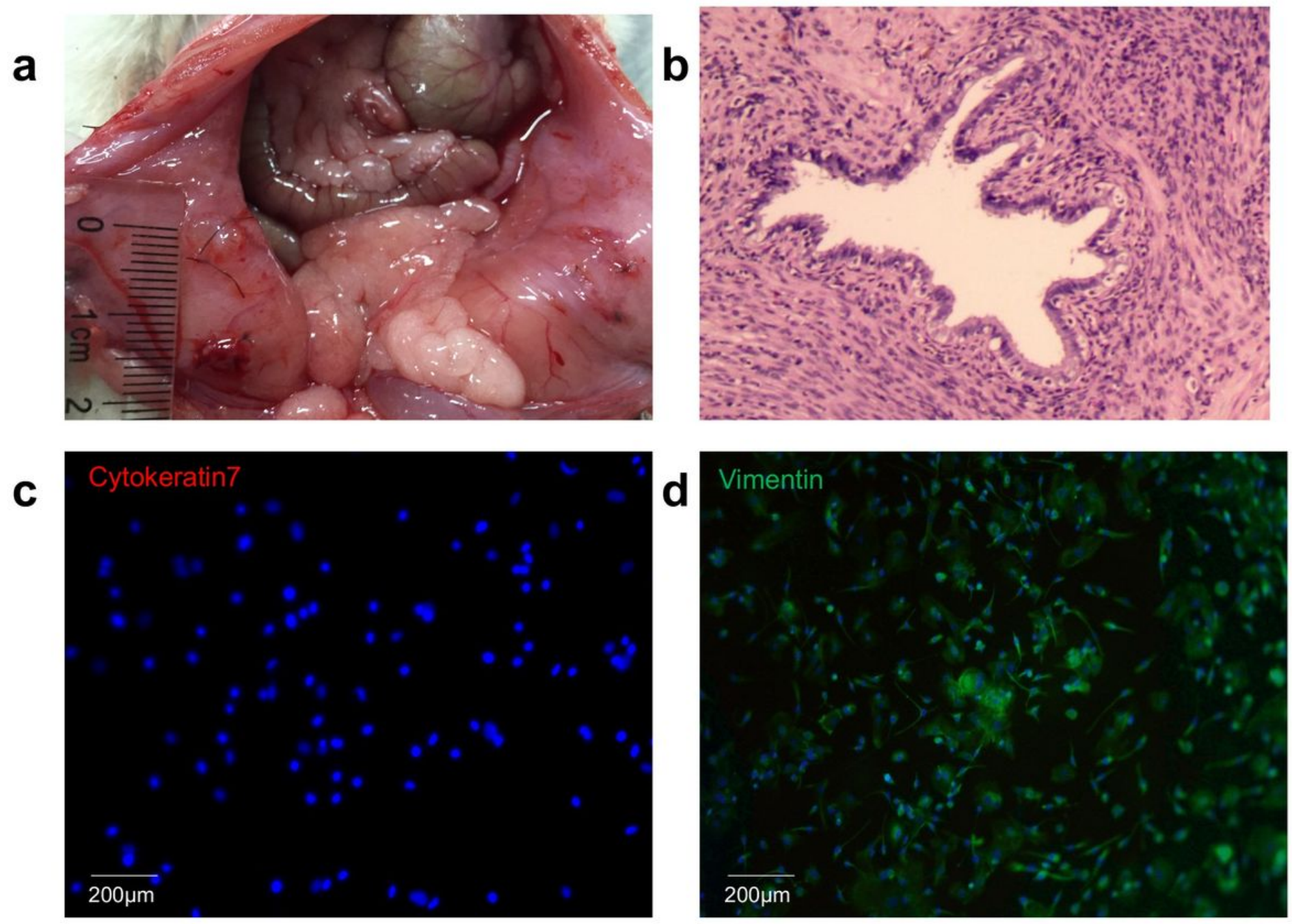

\section{Figure 2}

Identification of ESCs. (a) General observation of ectopic endometrial lesions. (b) Hematoxylin and eosin stain of ectopic lesion (magnification, x200). (c) ESCs were negative for Cytokeratin7. (d) ESCs were positive for Vimentin. Magnification, x100; Scale bar, $200 \mu \mathrm{m}$. ESCs, endometrial stromal cells. 
a

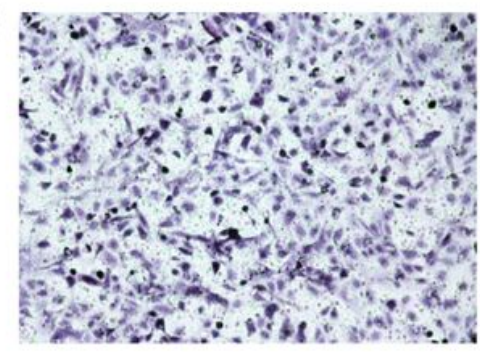

Control

b

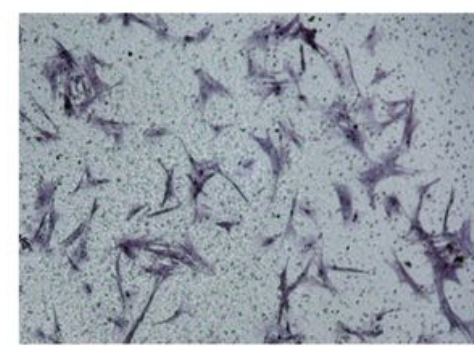

Control

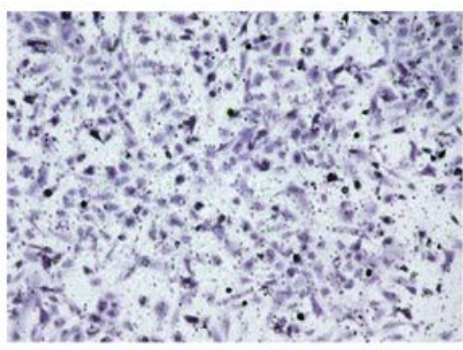

PCB
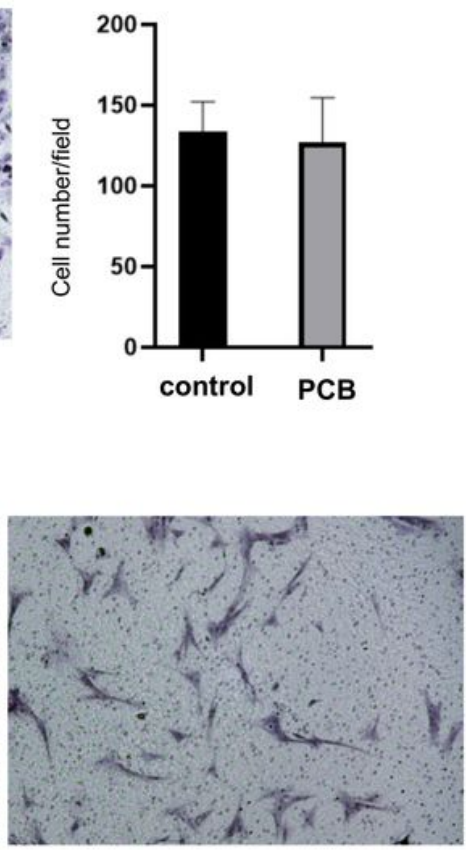

PCB+GSK126

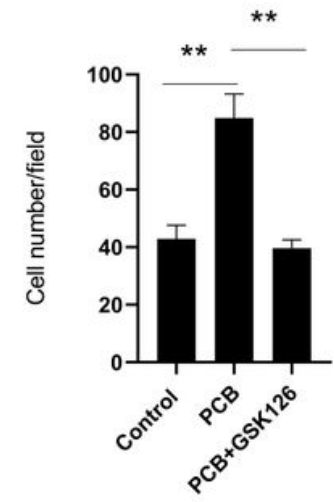

Figure 3

Effects of gestational PCBs exposure on ectopic ESC migration and invasion. (a) No difference was found in the migratory ability of the ESCs of the offspring. (b) Prenatal PCBs exposure promoted the invasion of offspring ectopic ESCs. Furthermore, GSK126, an inhibitor of enhancer of zeste 2 polycomb repressive complex 2 subunit, significantly decreased the invasive ability induced by PCBs. Magnification, $x 100$. ${ }^{\star *} P<0.01$. ESCs, endometrial stromal cells; PCBs, polychlorinated biphenyls. 


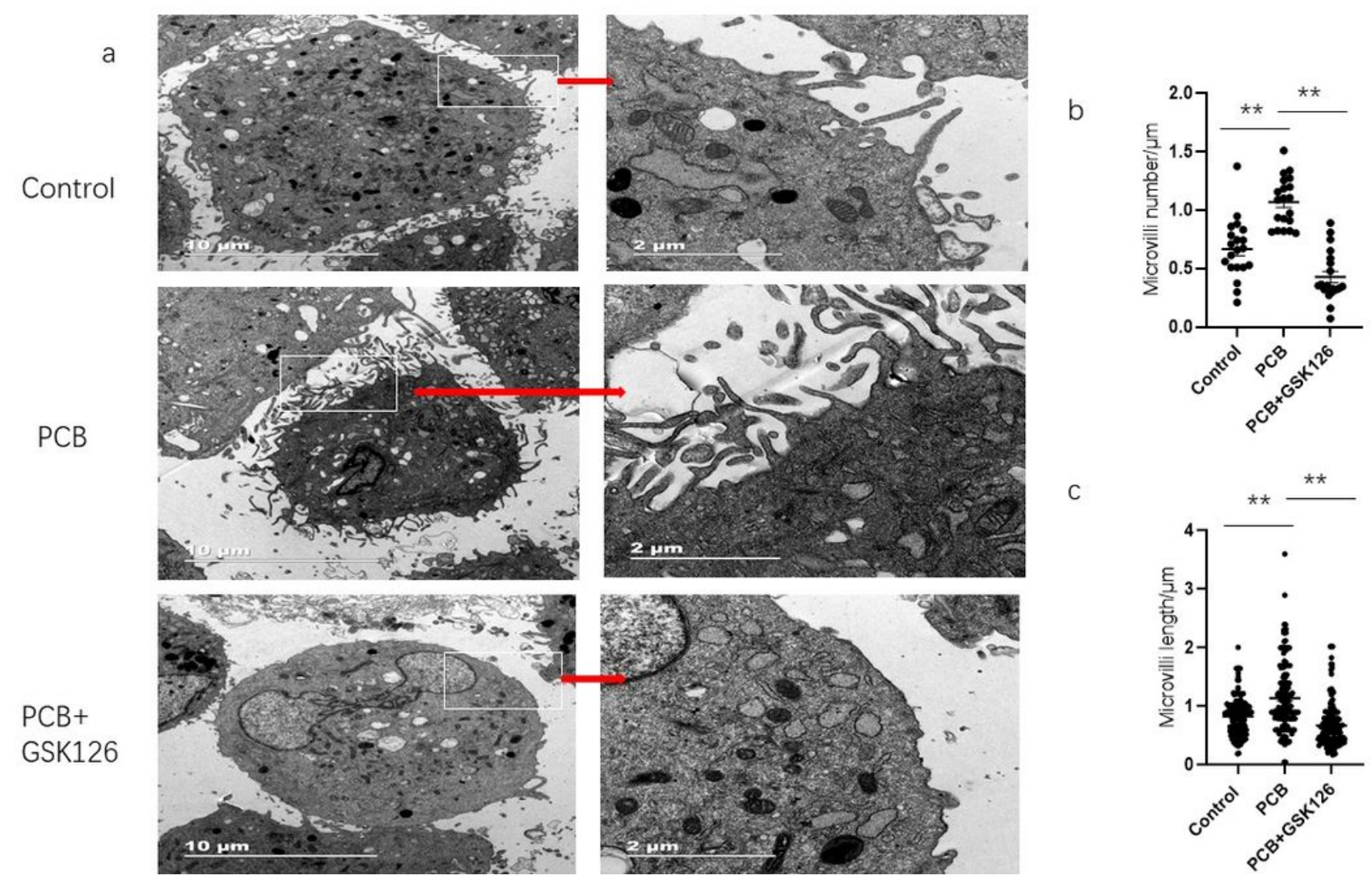

\section{Figure 4}

Ultrastructure of cell surface under transmission electron microscope. (a) Cell surface of ectopic ESCs from control group, PCB group and ectopic ESC in PCB group after EZH2 was inhibited by GSK126. (b) Analysis of microvilli number. (c) Analysis of microvilli length. a(left): Magnification, x4,800; a(right): Magnification, $x 18,500$. ${ }^{\star} \mathrm{P}<0.01$. ESCs, endometrial stromal cells; $\mathrm{PCBs}$, polychlorinated biphenyls; $\mathrm{EZH} 2$, enhancer of zeste 2 polycomb repressive complex 2 subunit. 

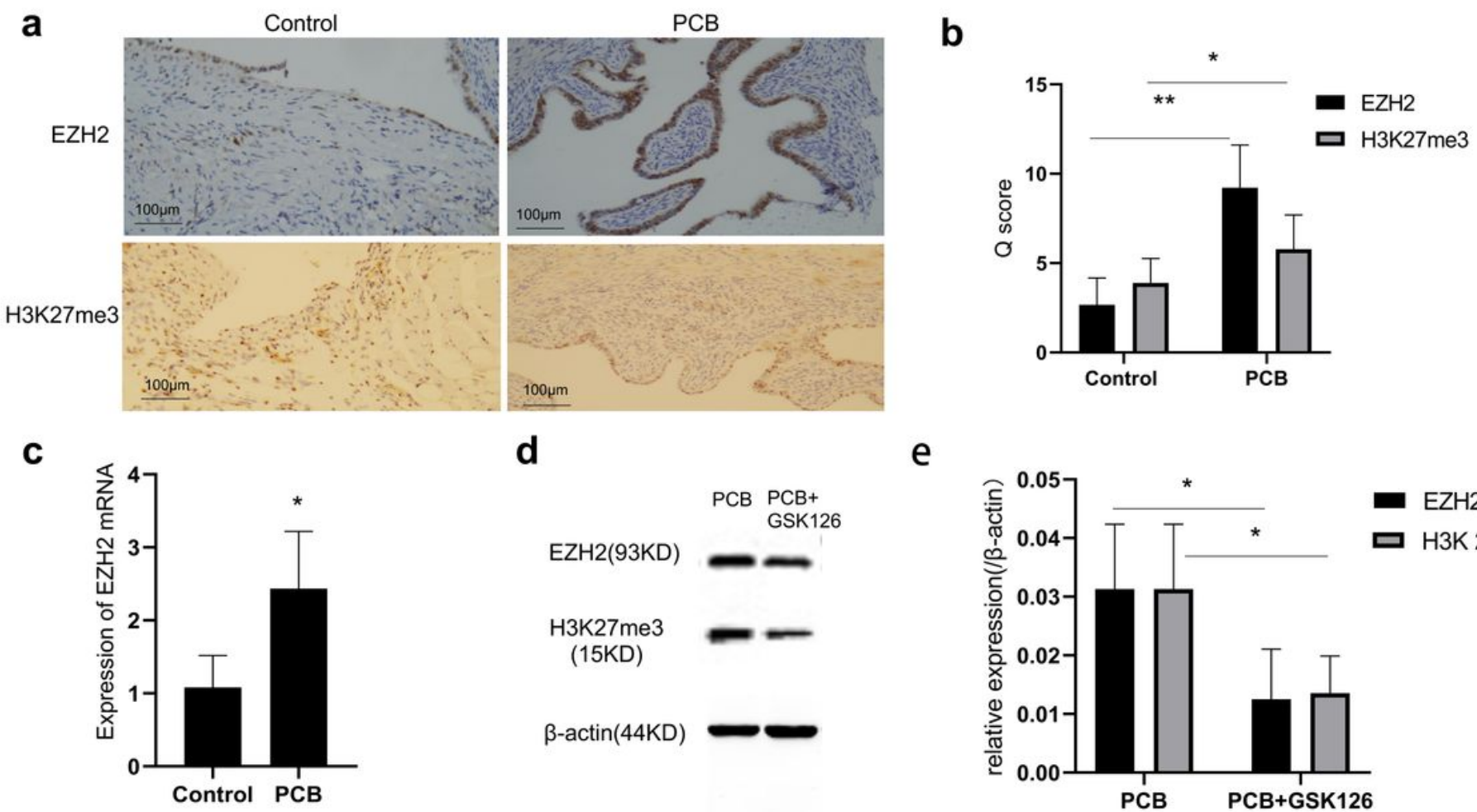

e

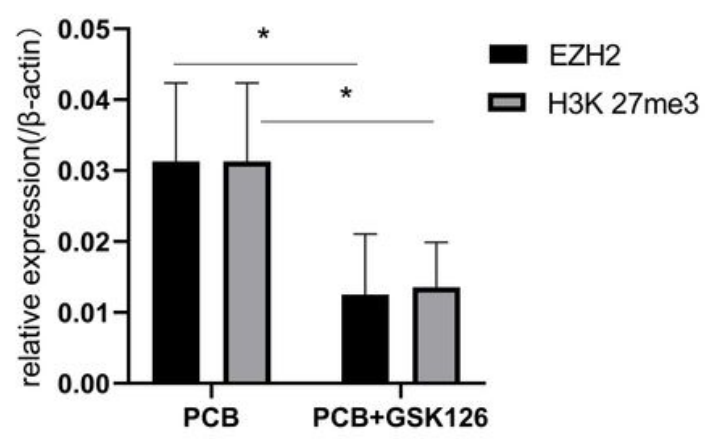

Figure 5

Expression levels of EZH2 and H3k27me3 in offspring ectopic ESCs. (a) Immunohistochemistry (magnification, x200; scale bar, $100 \mu \mathrm{m}$ ) in tissue sections shows EZH2 and H3k27me3 expression levels were increased after gestational PCBs exposure. (b) Graph representing the Q score for EZH2 and $\mathrm{H} 3 \mathrm{H} 27$ me3 expression levels in offspring ectopic ESCs. (c) Graph depicting the mRNA expression of EZH2 in offspring ectopic ESCs. (d) Western blotting results demonstrated that (e) EZH2 and H3k27me3 expression levels were abrogated by GSK126. ${ }^{*} P<0.05$, $* * P<0.01$. ESCs, endometrial stromal cells; PCBs, polychlorinated biphenyls; EZH2, enhancer of zeste 2 polycomb repressive complex 2 subunit; H3k27me3, trimethylation of Histone 3 lysine 27. 